\title{
Die distale Radiusfraktur - Konservative Behandlung
}

\author{
Nikolaus Schwarz
}

\section{Zusammenfassung}

Die Bedeutung der konservativen Therapie distaler Radiusfrakturen beschränkt sich nach heutiger Sicht auf Frakturen AO A2 und auf alte Patienten. Eine exakte Repositions- und Gipstechnik darf jedoch nicht in Vergessenheit geraten, weil damit häufig bessere Resultate erzielt werden, als ohne Einhaltung einer bewährten Technik. Auch mit der konservativen Therapie kann Schaden zugefügt werden ! Ob konservativ oder operativ behandelt werden soll, hängt in beson- derem Maße von den individuellen Gegebenheiten des Patienten ab und ist mit diesem abzustimmen. Reponiert wird am hängenden Unterarm unter Extension, gegipst wird in Funktionsstellung des Handgelenkes, d.h. $10^{\circ}$ Ulnarduktion, $5^{\circ}$ Extension. Gipsbefristung vier bis fünf Wochen. Wesentliche Basis einer erfolgreichen konservativen Therapie sind die laufende klinische und radiologische Überwachung des Patienten sowie eine sofort einsetzende Physikotherapie.

\section{Einleitung}

Alle distalen Radiusfrakturen können konservativ behandelt werden, fast alle derartigen Frakturen können reponiert werden, jedoch lässt sich nur ein Teil der Frakturen im Gips in reponierter Stellung halten. Zur Reposition der distalen Radiusfraktur bedarf es eines Längszuges, der ursprünglich durch einen Assistenten oder einen Schraubenzugapparat, später und heute noch durch das Aufhängen des Armes mithilfe so genannter Mädchenfänger ausgeübt wird (Abb.1). Es ist nicht möglich, diesen Zug über längere Dauer aufrecht zu erhalten. Das führt zur Redislokation und schränkt die Anwendbarkeit konservativer Methoden ein. Fernandez et al. [2] schreiben 1996: „extraartikuläre Frakturen mit wenig radiologischen Hinweisen auf Fragmentierung, mit weniger als $5^{\circ}$ bis $10^{\circ}$ dorsaler oder volarer Abkippung des distalen Fragmentes und mit weniger als wenig Millimetern messender radialer Verkürzung

OP-JOURNAL 2003; 19: 16-20

(c) Georg Thieme Verlag Stuttgart · New York

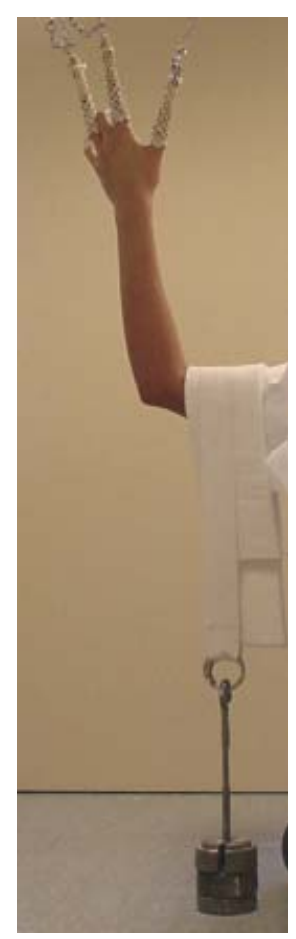

Abb.1 Aufhängen des Armes beim liegenden Patienten mit 90 gebeugtem Ellbogen, Fixation des Daumens, Sicherung an Zeige-, Mittel- und/oder Ringfinger und Extension mit 3 bis $5 \mathrm{~kg}$ für 5 bis 10 Minuten. sind stabile Frakturtypen. Nach erfolgter Reposition haben diese eine hohe Wahrscheinlichkeit, dass die Reposition durch Gipsfixation zu halten ist." In der Praxis sind solche Frakturtypen selten.

Ein gutes Repositionsergebnis geht manchmal schon nach 24 Stunden wieder verloren. Trotz minutiöser, wiederholt angewandter Repositions- und Gipstechniken heilen viele der konservativ behandelten Brüche in Fehlstellung aus. Gröbere Abweichungen der distalen Radiusgelenkfläche von der Norm, und hier vor allem die pathologische Neigung nach dorsal (Abb. 2), behindern die Funktion des Handgelenkes; Verkürzung des Radius bedingt eine relative Verlängerung der Ulna und damit eine schmerzhafte Kollision der Ulna mit der Handwurzel; Gelenkstufen (Abb.3) können wesentlich an der Entstehung einer $\mathrm{Ar}$ throse beteiligt sein, wenn dies auch nicht die einzig denkbare Ursache für Arthrose nach distaler Radiusfraktur ist. Deshalb fordern aktuelle Empfehlungen, dass eine Dorsalneigung des distalen Fragmentes weniger als $10^{\circ}$ und bleibende Gelenkstufen weniger als $3 \mathrm{~mm}$ betragen dürfen, um ein Behandlungsresultat bei ansonst idealen klinischen Bedingungen zu akzeptieren. Das distale Radio-ulnar-Gelenk muss zudem kongruent sein.

Es gilt also die Grundregel, dass bei distalen Radiusbrüchen die anatomische Wiederherstellung anzustreben ist. Immer dort, wo diese auf nicht operative Weise erreicht werden kann oder wo sich eine operative Therapie aus welchen Gründen auch immer verbietet, ist die konservative Therapie angezeigt.

\section{Indikation}

Die konservative Therapie der distalen Radiusfraktur ist bei stabilen extraartikulären Frakturen, vor allem AO Typ A2 [4] und bei Vorliegen von Kontraindikationen angezeigt. 

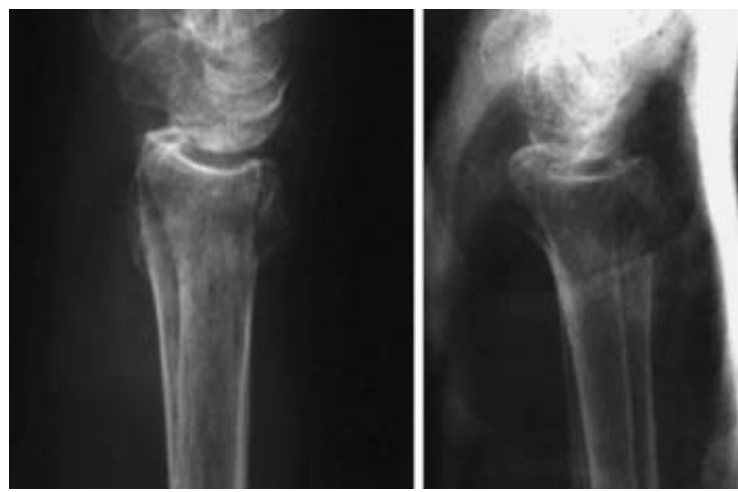

Abb. 2 96-jährige Patientin, einmalige Reposition der Fraktur am Unfalltag. Ausheilung mit der ursprünglichen Fehlstellung mit Neigung der distalen Radiusgelenkfläche nach dorsal. Ohne Reposition wäre dasselbe Ergebnis zu erwarten gewesen.
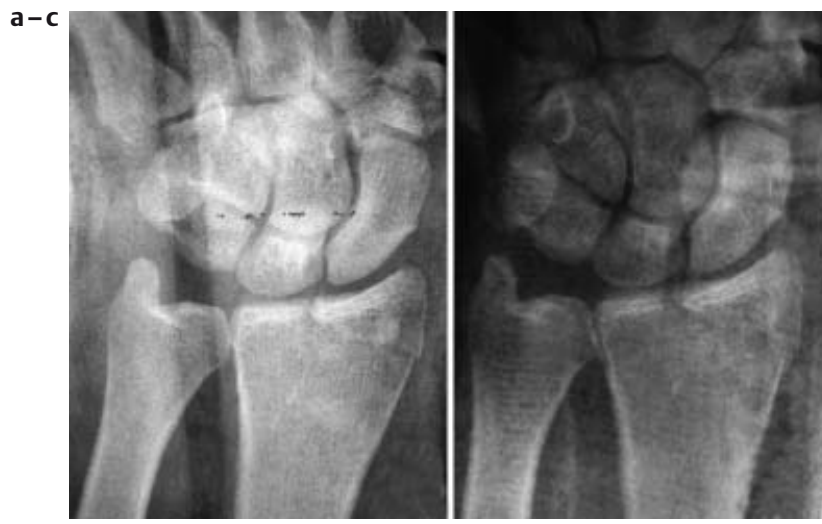

Abb.3 Mehrfach kontrollierte distale Speichenfraktur mit einer Gelenkstufe von maximal $1 \mathrm{~mm}$ (a), später von $2 \mathrm{~mm}$ (b). Die Stabilität war nicht richtig eingeschätzt und die sukzessive Dislokation nicht beachtet worden. Bei der Gipsabnahme zeigte sich eine reparationsbedürftige Gelenkstufe von rund $4 \mathrm{~mm}$ (c).

Stabile Frakturen haben eine Fehlstellung am ersten Röntgenbild, die $20^{\circ}$ dorsale Neigung nicht überschreitet, sie haben keine dorsale Trümmerzone, sie weisen keine Fraktur der Gelenkfläche auf und die Patienten sind jünger als 60 Jahre.

Instabile Frakturen haben $>20^{\circ}$ dorsale Neigung, eine dorsale Trümmerzone, einen Ulnavorschub von $>3 \mathrm{~mm}$, intraartikuläre Frakturen, ulnare Begleitverletzungen, sowie ein Patientenalter von mehr als 60 Jahren. Treffen drei oder mehr der genannten Kriterien $\mathrm{zu}$, ist von einer instabilen Situation auszugehen.

Distale Speichenfrakturen sind Brüche des osteoporotischen Knochens beim älteren und alten Patienten. Deshalb sind Kontraindikationen für operative Eingriffe mit relativer Indikation häufig zu finden. Auch sind die Ansprüche der Patienten an die Funktion des Handgelenkes stark unterschiedlich.
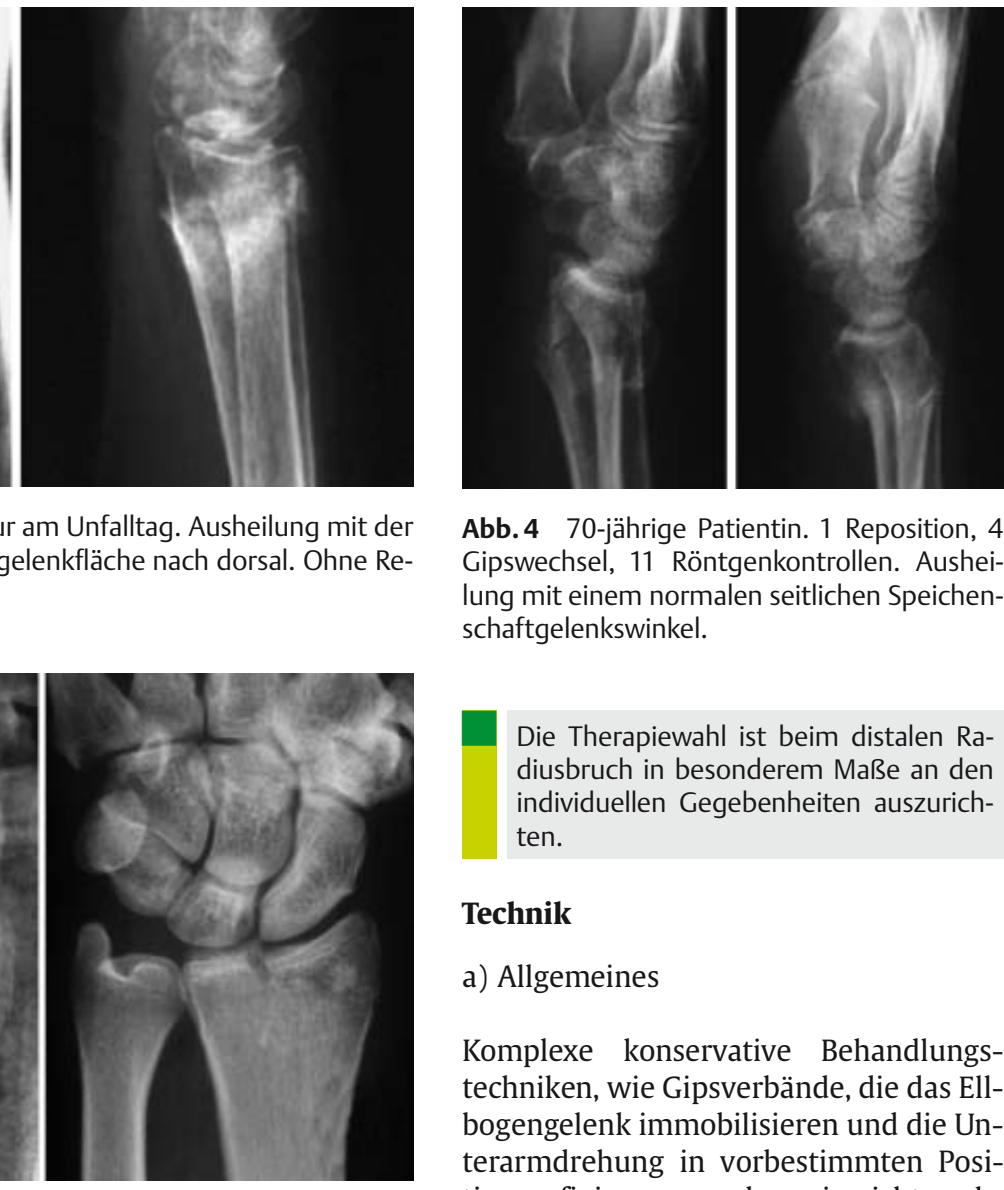

Abb. 4 70-jährige Patientin. 1 Reposition, 4 Gipswechsel, 11 Röntgenkontrollen. Ausheilung mit einem normalen seitlichen Speichenschaftgelenkswinkel.

Die Therapiewahl ist beim distalen Radiusbruch in besonderem Maße an den individuellen Gegebenheiten auszurichten.

\section{Technik}

\section{a) Allgemeines}

Komplexe konservative Behandlungstechniken, wie Gipsverbände, die das Ellbogengelenk immobilisieren und die Unterarmdrehung in vorbestimmten Positionen fixieren, wenden wir nicht mehr an. Entweder operieren wir diese Patienten oder aber es verbietet sich aus welchen Gründen auch immer eine Operation und damit auch eine schwierige und belastende Gipsbehandlung. In diesen Fällen wird im Unterarmgipsverband behandelt (Abb.5) und es wird je nach den Umständen entschieden, ob eine Reposition der Fragmente durchgeführt wird oder auch auf die Reposition verzichtet wird. Das betrifft dann vor allem alte, morbide Patienten.

Die in der Folge beschriebene Technik der konservativen Behandlung bezieht sich auf eine der möglichen Varianten, wie sie von Lorenz Böhler [1] entwickelt und zuletzt von Jahna et al. [3] beschrieben worden war. Sie beruht auf der Reposition im Längszug und auf der Gipsruhigstellung in Funktionsstellung, also in $10^{\circ}$ Ulnarduktion und $5^{\circ}$ bis $10^{\circ}$ Überstreckung des Handgelenkes. tienten, seine Erwartungen und Bedürfnisse ebenso miteinzubeziehen, wie medizinische und soziale Aspekte. Die Entscheidung für oder gegen eine Operation ist sehr individuell zu stellen.

Stabile, extraartikuläre distale Radiusfrakturen vom AO-Typ A2 eignen sich zur konservativen Behandlung.
Die Ruhigstellung des Handgelenkes erfolgt in $10^{\circ}$ Ulnarduktion und $5^{\circ}$ bis $10^{\circ}$ Überstreckung.

Diese Grundsätze sind nicht allgemein akzeptiert. Nach anderen Schulen erfolgt die Reposition durch Palmarkippung des 


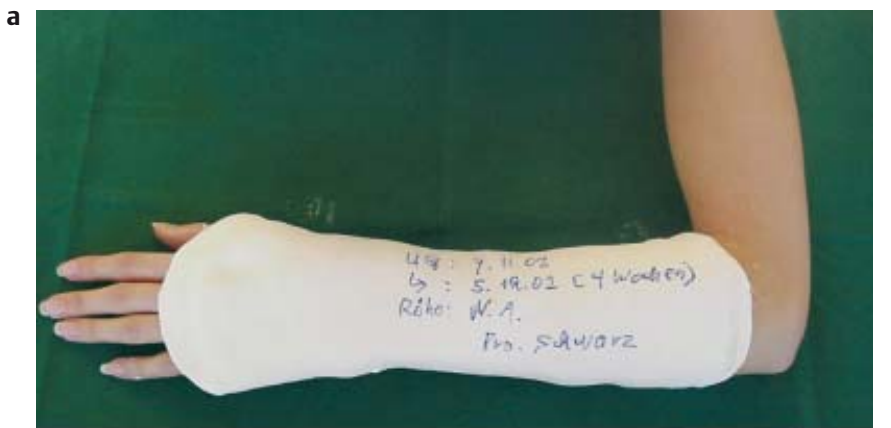

b
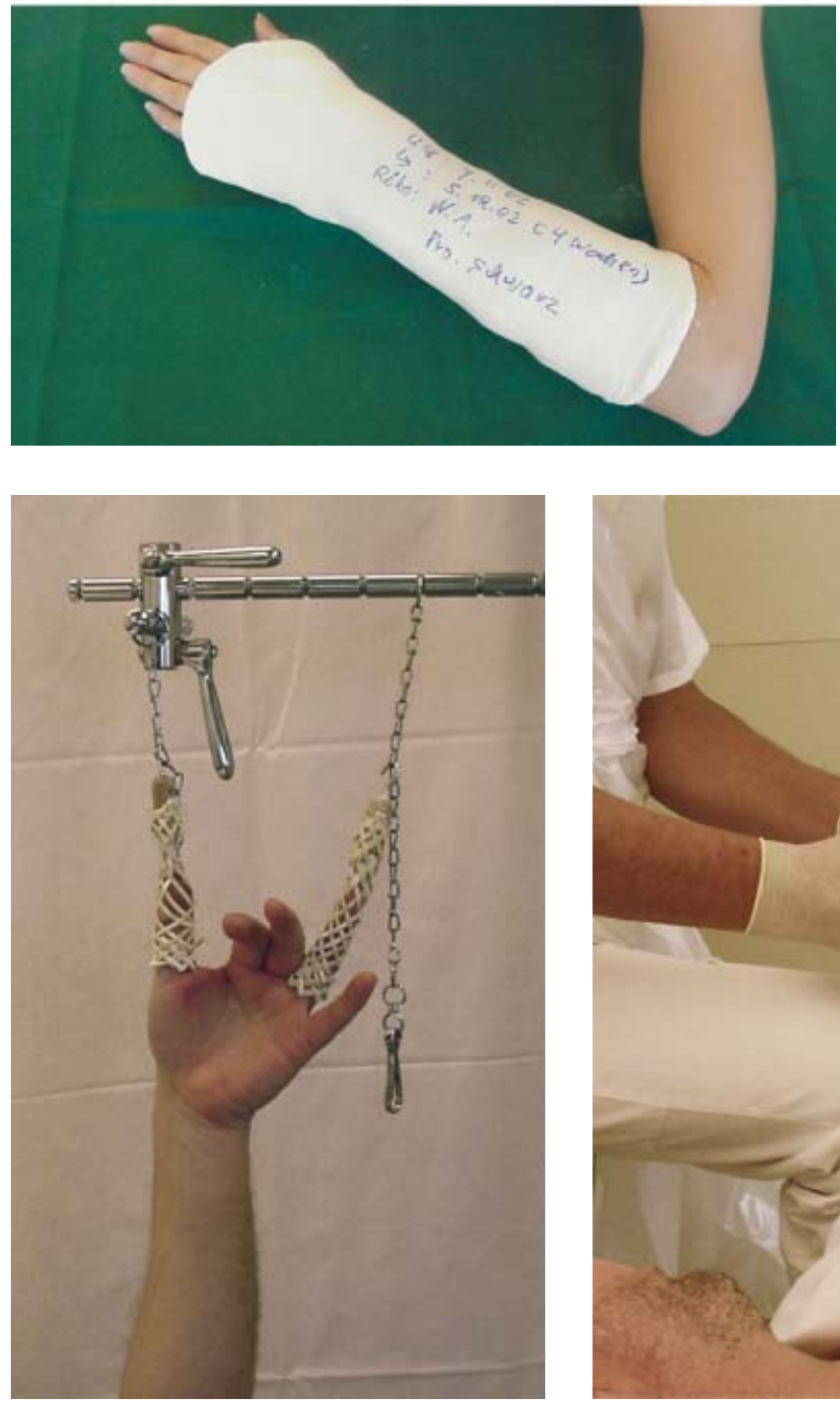

Abb.6 Aufhängung des Daumens im Mädchenfänger und Sicherung am Ringfinger.
Abb. 5 Typische dorsale Gipsschiene mit Beschriftung (a). Der Gips behindert die Beugung des Ellbogengelenkes nicht (b).

Handgelenkes, etwa über eine Tischkante oder durch Manipulation, und die Fixation erfolgt in $10^{\circ}$ bis $15^{\circ}$ Beugestellung [2]. Während mit beiden Methoden gleich gute Repositionsresultate zu erzielen sind, liegt der Vorteil der Palmarkippung in einer angeblich besseren Retention der Fragmente, die Nachteile sind hartnäckige Bewegungsbehinderungen des Handgelenkes und fallweise auch Störungen des Nervus medianus.

Die distalen Radiusfrakturen benötigen 4 bis 6 Wochen zur knöchernen Heilung.

b) Durchführung

- Zur Reposition der Fraktur erfolgt eine Schmerzausschaltung vorzugsweise in Lokalanästhesie. Dazu werden rund $5 \mathrm{ccm} \mathrm{1-2 \%} \mathrm{Lokalanästhetikum} \mathrm{unter}$ sterilen Bedingungen in das Frakturhämatom injiziert. Der Wirkungseintritt ist nach etwa 10 Minuten zu erwarten.

- Der Daumen wird mit einem so genannten Mädchenfänger gefasst und so aufgehängt, dass der Ellbogen $90^{\circ}$ gebeugt ist und der Arm in der Schulter $90^{\circ}$ abduziert ist (Abb.1). Weitere Mädchenfänger sichern Zeige-, Mittel- und/oder Ringfinger (Abb.6). An einer um den Oberarm geführten gepolsterten Schlaufe werden 3 bis $5 \mathrm{~kg}$ Extensionsgewicht angehängt (Abb.1). Der Zug soll ungestört über 5 bis 10 Minuten wirken.

- Die Reposition erfolgt durch Ulnarduktion und anschließend Beugung im Handgelenk sowie durch direkten Druck auf das distale Fragment (Abb. 7).

a Nun wird eine feuchte, breite Gipslonguette dorsalseitig angelegt. Sie muss aber auch die Beugeseite der Speiche umfassen. Anwickeln mit einer feuchten Mullbinde (Abb.8).

- Während die Gipslonguette trocknet, wird sie anmodelliert: streckseitig über distalem Fragment und Handgelenk (Abb.11 b) sowie volarseitig zentral der Fraktur.

- Die Mullbinde wird nach dem Trocknen durchgespalten und die Gipsränder werden aufgebogen (Abb.8). Mit einer trockenen Mullbinde wird erneut überwickelt. Beschriftung des Gipsverbandes: Unfalldatum, Gipsdatum, Befristung des Gipsverbandes, Datum der nächsten Röntgenkontrolle, Name des Arztes (Abb.5).

- Es bleibt zu beachten, dass die Hohlhandtour nicht aus Gips und nicht zu eng sein darf, um die Fingerbeugung nicht zu beeinträchtigen (Abb.9). Weiters ist sicherzustellen, dass der Gips- 
a, b
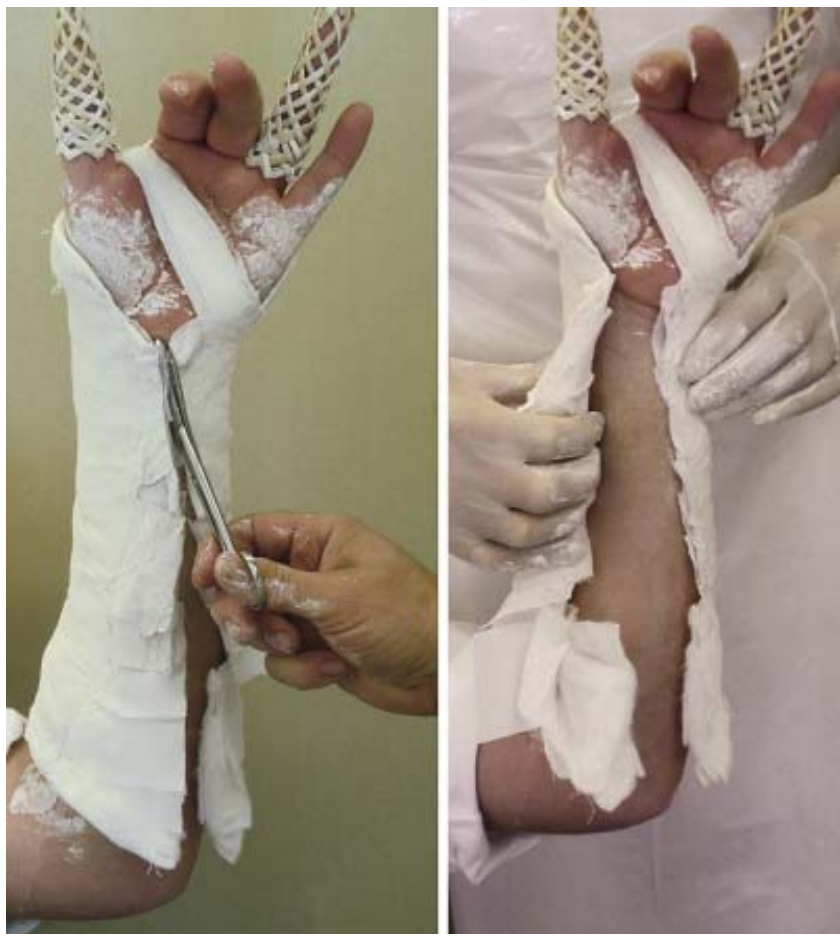

Abb. 8 Die dorsale Gipslonguette wurde mit einer feuchten Mullbinde angewickelt. Diese wird bis auf den letzten Faden durchgespalten (a) Die Gipsränder werden aufgebogen, um zu verhindern, dass sie Druck auf die Weichteile ausüben. Man sieht auf dieser Aufnahme, wie weit die dorsale Gipslonguette an die Beugeseite der Speiche reicht (b).
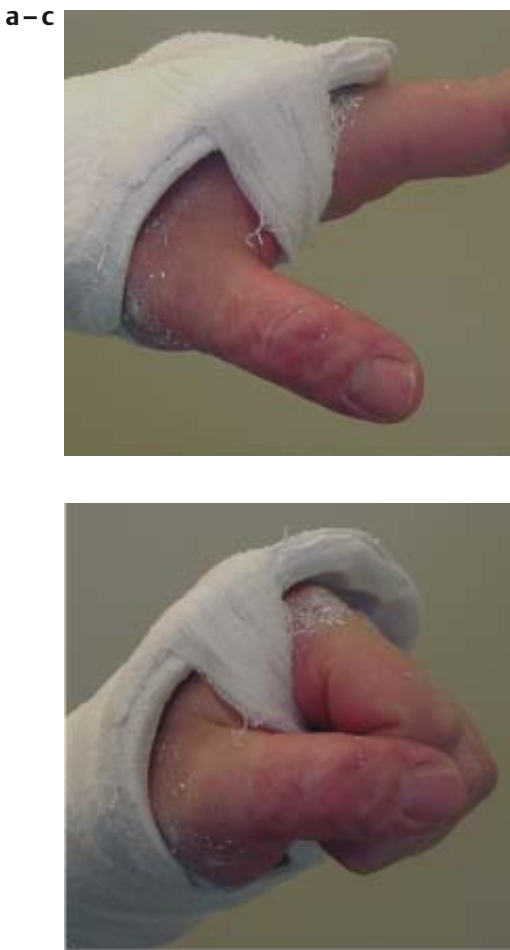

Abb. 9 Freie Streckung der Finger bei leichter Dorsalextension des Handgelenkes (a). Freier Faustschluss (b). Die Beugung nicht behindernde Hohlhandtour mittels Mullbinde (c).
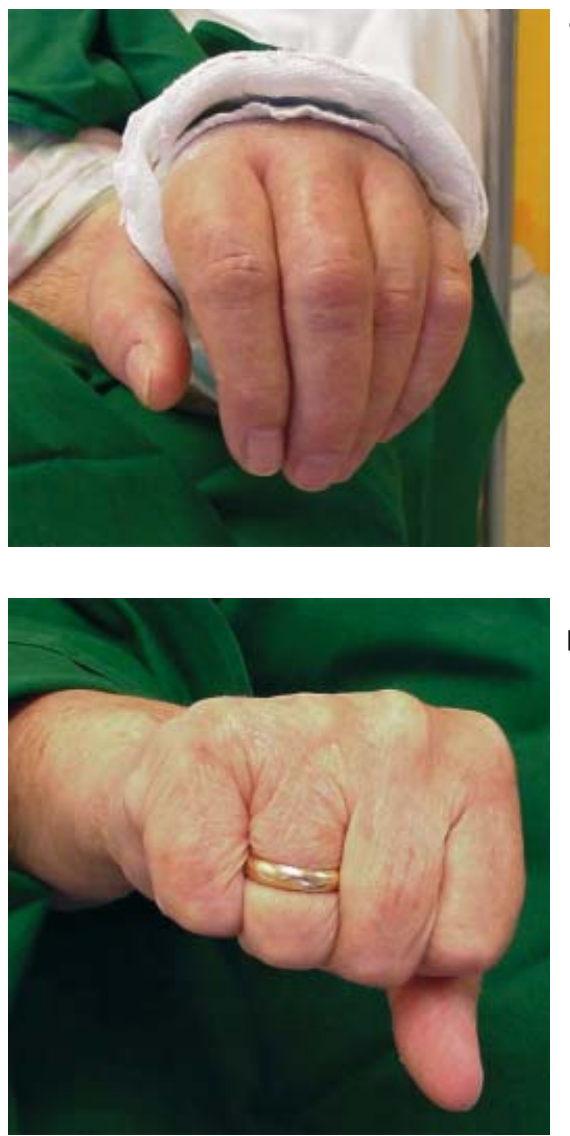

Abb.10 Die Gipslonguette ist über der Mittelhand stark nach dorsal konvex gekrümmt. Dadurch wird der Faustschluss, den der Patient bei dieser Aufnahme versucht, undurchführbar (a), weil beim Faustschluss die Mittelhandköpfchen in einer Reihe nebeneinander stehen (b) und dieser nur möglich ist, wenn sie dazu ausreichend Platz haben.

Behandlungsschema konservativ behandelter distaler Radiusfrakturen Tag 0: Reposition dorsale Gipsschiene für den Unterarm (beschriften!) Röntgenkontrolle Übungen und Funktionen zeigen Analgetika

Tag 1: Zirkulationskontrolle Funktionskontrolle zirkuläres Schließen des Gipsverbandes mit Gips (beschriften!) Anweisung oder Zuweisung zur Physikotherapie Bestehen Bedenken bezüglich der Qualität von Reposition und Retention, ist eine frühzeitige Röntgenkontrolle nach 24 bis 48 Stunden angezeigt.
Tag 7: Funktionskontrolle Röntgenkontrolle Gipswechsel unter Extension

Tag 14: Funktionskontrolle Röntgenkontrolle ggf. Gipswechsel

Tag 21: Funktionskontrolle ggf. Röntgenkontrolle ggf. Gipswechsel

Tag 28 oder Tag 35: Gipsabnahme Röntgenkontrolle elastische Binde Physikotherapie

Untersagt sind Massagen und warme oder lauwarme Handbäder, erlaubt ist Lymphdrainage! 
verband die Ellbogenbeugung nicht behindert (Abb.5), sowie dass der Gips über dem Handrücken nicht konvex, sondern plan ist, um den Faustschluss zu ermöglichen (Abb.10).

Frühzeitige Physikotherapie hat auch bei der konservativen Therapie einen wesentlichen Anteil am Therapieerfolg

Massagen sowie Handbäder sind nicht nur entbehrlich, sondern schädlich.

\section{Komplikationen}

Das Complex Regional Pain Syndrome (CRPS), in der Praxis nach wie als Morbus Sudeck bezeichnet, ist eine wiederholt zu beobachtende Komplikation auch nach konservativ behandelten distalen Radiusfrakturen. Die Krankheitsursache ist ungeklärt. Erfahrungsgemäß gilt Schmerz als ein möglicher Auslöser, weshalb mehrfache Repositionsversuche die Entstehung des Syndroms fördern sollen. Ebenso besteht der Verdacht, dass zu enge Gipsverbände oder fehlende aktive Bewegungen der nicht fixierten Gelenke an der Auslösung beteiligt sind. Nahezu regelmäßig findet man das CRPS an der Hand nach Anwendung von Massagen und warmen Bädern; eine Praxis, die trotz jahrzehntelanger Warnungen immer noch ihre Anhänger hat.

Durch Gipsdruck kann es zu einer Störung der Ausläufer des Nervus radialis superficialis und zu Parästhesien über der radialen Mittelhand kommen. Es genügt, den Patienten über die passagere Art der Störung zu informieren.
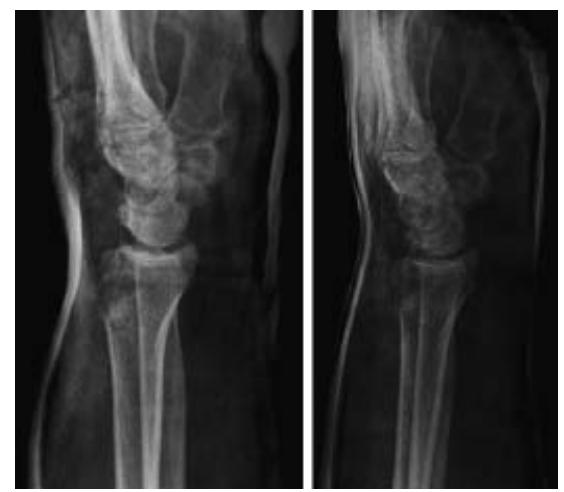

Abb.11 Die Delle über dem distalen Speichenfragment ist zu weit proximal und kann Druckulzera verursachen (a). Korrekte Delle und gut anmodellierter Gipsverband (b).

Ebenfalls durch Gipsdruck, vor allem bei falscher Modellierung (Abb.11), können Hautulzera entstehen, wobei das bei der häufig atrophen Haut der Patienten nicht nur sehr leicht passiert, sondern meist auch keinerlei Schmerzen verursacht.

In weniger als $1 \%$ der Patienten kommt es im Verlauf der ersten Wochen nach der Fraktur zu einem Riss der Sehne des langen Daumenstreckers, wobei das vom Patienten manchmal nicht sofort bemerkt wird. Die Therapie besteht in einer Sehnenersatzoperation mit der Sehne des Musculus extensor indicis proprius.

Ebenfalls unter Komplikation zu erwähnen ist die Fehleinschätzung der Klassifikation der Fraktur und deren Stabilität (Abb.3). Eine solche Fehlklassifikation bedingt ggf. einen frühzeitigen Stellungsverlust, der zu einem Überdenken der Therapie Anlass geben muss. Bei so genannten konservativen Behandlungsversuchen ist ein Therapiewechsel auf ein operatives Verfahren nicht nur dann zu prüfen, wenn es zu einem raschen Repositionsverlust kommt. Vielmehr sind jene schleichenden Repositionsverluste im Auge zu halten, bei welchen von einer Röntgenkontrolle zur nächsten immer nur tolerable $3^{\circ}$ oder $5^{\circ}$ Verlust eintreten, diese sich aber letztlich $\mathrm{zu}$ einem schlechten radiologischen Ergebnis summieren. Es muss deshalb immer mit dem Röntgenbild der ersten Reposition verglichen werden.

\section{Literatur}

1 Böhler L. Die Technik der Knochenbruchbehandlung. Maudrich Wien 1957

2 Fernandez DL, Jupiter JB. Fractures of the distal radius. Springer Berlin 1996

3 Jahna H, Wittich H. Konservative Methoden in der Frakturbehandlung. Urban und Schwarzenberg Wien 1985

${ }^{4}$ Oestern HJ. Behandlung der distalen Radiusfraktur. In: KP Schmit-Neuerburg, H Towfigh, R Letsch (Hrsg) Tscherne Unfallchirurgie - Ellbogen, Unterarm Springer Berlin $2001 ; 225-248$

Univ.-Prof. Dr. Nikolaus Schwarz Facharzt für Unfallchirurgie und Sporttraumatologie

Ärztlicher Leiter

Unfallkrankenhaus Klagenfurt

Waidmannsdorferstr. 35

A-9020 Klagenfurt 